\title{
Can a Bicycle Create a Unicycle Track?
}

\author{
David L. Finn
}

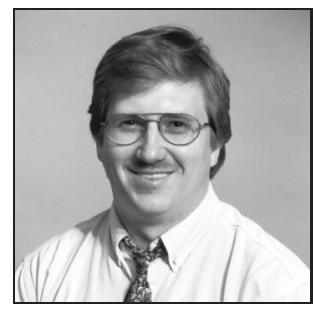

David L. Finn (david.finn @ rose.hulman.edu) is an assistant professor of mathematics at Rose-Hulman Institute of Technology. He has a B.S. in applied mathematics from Steven Institute of Technology and a M.S. and Ph.D. in mathematics from Northeastern University. His current mathematical interests lie in the interplay of analysis, geometry, and physics. His non-mathematical interests include his family, specifically his wife and new-born baby, and rooting for the Red Sox.

\section{Introduction}

In the Sherlock Holmes mystery "The Priory School," a telling piece of evidence comes from the observation and ensuing analysis of a pair of tire tracks. This evidence led Holmes and Watson to discover the murder of a school master, and subsequently to discover the identity of a Duke's son. The critical part of the analysis is Holmes' argument that the direction a bicycle was traveling can be determined by using the indentation of the tire tracks at a crossing to determine which track was created by the back tire. Once this is known, the direction the bicycle was travelling can be determined by the orientation of the treads of the back tire. Problems have been noted with Holmes' argument (see [9]). However, there are other methods for determining the direction a bicycle was travelling. For instance, in the solution to the title problem of the book Which way did the bicycle go? [9], the authors show how the direction a bicycle is traveling can be determined using a simple calculus argument.

We consider the following variation of the Holmes mystery: Imagine that Holmes and Watson discover a single tire track, as in Figure 1, instead of a pair of tire tracks, as in Figure 2, while walking down a path at the Priory School. How would Holmes and Watson determine which way the cyclist was heading? In this situation, it seems natural to suspect that Watson would come to the obvious conclusion that the track was created by a unicycle, and thus the direction the cyclist was heading cannot be determined. However, we suspect that Holmes might have drawn on his famous question to Watson,

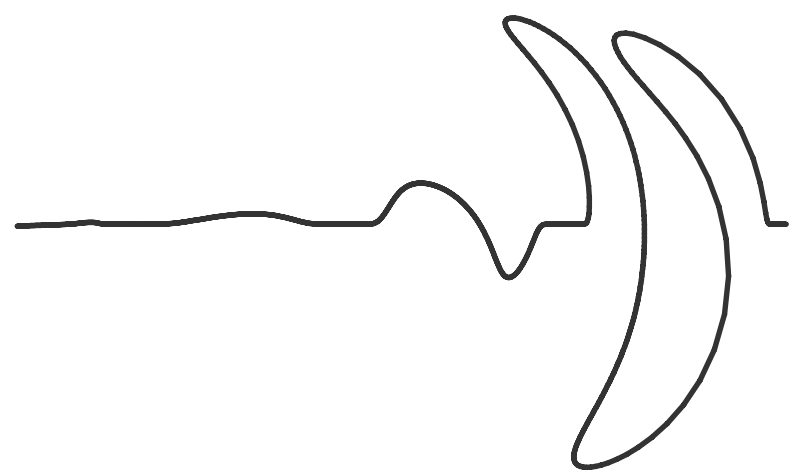

Figure 1. Can a bicycle create this track? 


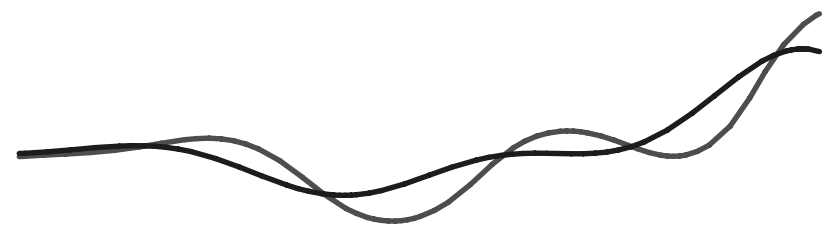

Figure 2. A pair of tire tracks

"How often have I said to you that when you have eliminated the impossible, whatever remains, however improbable, must be the truth?," to ask our title question.

Most of the people that we asked, "Can a bicycle create a single tire track?", have immediately answer $N O$, at least once we have eliminated the possibility of a single straight tire track. A few people suspect that we have asked them a trick question, and say a skilled rider can ride a bicycle like a unicycle by keeping the front tire in the air (a wheelie). Once this possibility has been eliminated, and we explain that both tires must remain in contact with the ground at all times, the answer seems to be emphatically, NO. A very few still suspect that this is a trick question, but they cannot find the trick. These last few are of course correct, but the trick involves nothing other than mathematics.

Our goal in this paper is to provide a convincing argument to anyone with a solid understanding of calculus that it is possible to create a single tire track with a bicycle. We will not provide the formal existence proof here, as it involves establishing the existence of a global solution to a differential difference equation. Here, we use a more geometrical approach combined with a numerical construction. Our hope is that after reading this article one will be able to understand and alter the numerical algorithm provided in Section 6 and on a Maple worksheet at the author's web page [6] to produce other examples of unicycle tracks that can be created a bicycle.

\section{Physical constructions}

Rather than starting with the mathematical construction, we first describe a physical method for constructing a unicycle track with a bicycle. This construction method is based on the same method for checking whether a bicycle can construct a given set of tire tracks. To describe the method, we represent the given unicycle track with a parametric curve $\gamma$ parameterized with respect to its arclength $s$. We will describe the tire tracks of a bicycle with a pair of parametric curves $\alpha$ and $\beta$ that we will parameterize with respect to time, where $\alpha(t)$ and $\beta(t)$ represents the position of the point of contact of the front and back tires and the ground at time $t$, respectively.

Our physical check for whether a bicycle can follow a unicycle track requires that we be able to place both tires of the bicycle on the unicycle track at the same time. In addition, we must be able to push the bicycle on the unicycle track keeping both bicycle tires in contact with the unicycle track at all times. This means that for each time $t$ there must exist positions $\gamma\left(s_{1}(t)\right)$ and $\gamma\left(s_{2}(t)\right)$ on the unicycle track such that $\beta(t)=\gamma\left(s_{1}(t)\right)$ and $\alpha(t)=\gamma\left(s_{2}(t)\right)$. We can now check whether a bicycle can create a unicycle track by first checking whether we can place the bicycle on the unicycle track at time $t=0$, i.e., do there exist points $\gamma\left(s_{1}(0)\right)$ and $\gamma\left(s_{2}(0)\right)$ such that $\beta(0)=\gamma\left(s_{1}(0)\right)$ and $\alpha(0)=\gamma\left(s_{2}(0)\right)$ ? Next, we push the bicycle on the unicycle track in such a manner that the front tire remains in contact with the unicycle track, i.e., $\alpha(t)=\gamma\left(s_{2}(t)\right)$ for some function $s_{2}(t)$. If it happens that the back tire remains in contact with the unicycle track, i.e., $\beta(t)=\gamma\left(s_{1}(t)\right)$ for some function $s_{1}(t)$, then the 
unicycle track could have been created by a bicycle. We note that we could have just as easily pushed the bicycle on the unicycle track so that the back tire remains in contact with the unicycle track and tested whether the front tire track remains in contact with the unicycle track.

Our physical construction method is based on the following variation of the physical check. Start with a segment of a unicycle track $\gamma(s)$ with $s_{1}<s<s_{2}$, and suppose we can place the bicycle on this track segment with $\alpha(0)=\gamma\left(s_{2}\right)$ and $\beta(0)=\gamma\left(s_{1}\right)$. Now, push the bicycle forward on the curve segment keeping the back tire of the bicycle in contact with the unicycle track, and extend the segment of the unicycle track by constructing $\alpha(t)$ from $\beta(t)$. Next, push the bicycle backward on the curve segment keeping the front tire of the bicycle in contact with the unicycle track, and extend the segment of the unicycle track by constructing $\beta(t)$ from $\alpha(t)$. This process of pushing the bicycle forward or backward on the unicycle track may be continued indefinitely, provided the front or back tire can remain in contact with the curve segment created. This will be possible if the initial curve segment can be extended in an infinitely smooth manner by pushing the bicycle forward and backward. Therefore, given a suitable initial segment, we can create a unicycle track with a bicycle. This is of course provided we can determine the position of the front tire given the back tire track, and the position of the back tire given the front tire track.

The remainder of this paper describes this physical construction in mathematical terms. This requires an analysis of the geometry of bicycle tracks, which provides us with the equations necessary to mathematically construct a bicycle track and the relations necessary to construct an initial curve segment to start the construction process. We want to emphasize that constructing the initial curve segment is the difficult part of our construction, as we need the initial segment to satisfy an infinite number of conditions. If we apply our construction process to a generic initial curve segment, we can not continue the process indefinitely. For instance, applying our construction to the curve segment $\mathbf{c}(t)=\left[t, t^{2}(1-t)^{2}\right]$ with $0 \leq t \leq 1$ produces the curve in Figure 3 . Notice that the curve in Figure 3 is not smooth. Specifically, the curve ceases to be smooth at the point where the segment formed by pushing the bicycle forward meets the initial segment, which means that we cannot continue to push the bicycle forward any farther without destroying the continuity of the track.

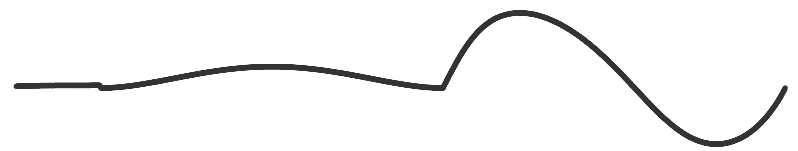

Figure 3. Curve generated with initial track $c=\left[t, t^{2}(1-t)^{2}\right]$

\section{Geometry of bicycle tracks}

Our construction process requires that we be able to generate the front tire track from the back tire track, and generate the back tire track from the front tire track. Generating the tire tracks in this manner entails deriving equations which relate the positions and unit tangent vectors of the front and back tire tracks, and their curvatures. To state these equations, we will need to use some elementary differential geometry, and some basic facts about bicycles. Most of the differential geometry that we need can be found in calculus textbooks, for instance [4] or [11]. For more complete details on the subject of differential geometry, see [2], [8] or [10]. 
We will assume for the rest of this paper that the bicycle is ridden on a perfectly flat surface, which means $\alpha, \beta$, and $\gamma$ will be plane curves. We will also assume the plane of each bicycle tire meets the ground (the surface on which the bicycle is ridden) in a right angle. These assumptions are imposed to simplify the analysis. However, we suspect that it is possible to construct a unicycle track with a bicycle using similar methods, but the equations involved in the construction process will be much more complicated.

Using our simplifying assumptions, the positions of the tires $\alpha(t), \beta(t)$ are related by

$$
\alpha(t)=\beta(t)+l \mathbf{T}_{\beta}(t),
$$

where $l$ is a positive constant representing the length of the bicycle and $\mathbf{T}_{\beta}(t)$ is the unit tangent vector of the back tire track $\beta$ at time $t$. This equation arises from two observations about how a bicycle is built. The first observation is that the back tire is fixed in the frame, meaning that the back tire and the frame are aligned. Thus, the tangent line to the back track at time $t$ is equal to the secant line through the front and back tire positions at time $t$, i.e.,

$$
\mathbf{T}_{\beta}(t)=\frac{\alpha(t)-\beta(t)}{\|\alpha(t)-\beta(t)\|} .
$$

The second observation is that the frame is rigid, meaning that the distance $l=\|\alpha-\beta\|$ between the points of contact between the tires and the ground is a constant. To simplify the remaining equations, we will take $l=1$.

For convenience, we will denote the intrinsic geometric properties of the curves $\alpha, \beta$ and $\gamma$ by using subscripts. The unit tangent of $\alpha$ will be denoted by $\mathbf{T}_{\alpha}$, the unit tangent of $\beta$ will be denoted by $\mathbf{T}_{\beta}$, and the unit tangent of $\gamma$ will be denoted by $\mathbf{T}_{\gamma}$. When there is no subscript, we are referring to quantities of a generic plane curve. Also, we will usually suppress the dependence of all quantities on the parameters, with the exception being when we differentiate. Then, we will use differential notation, $d \mathbf{T} / d s$ or $d \mathbf{T} / d t$.

It is worth noting that while riding a bicycle one does not have direct control of the back tire's position or direction. One has direct control over the direction of the front tire (at least with respect to the frame). This means one has direct control over the angle $\Theta$ between the front tire track's unit tangent vector $\mathbf{T}_{\alpha}$ and the frame (the back tire track's unit tangent vector) $\mathbf{T}_{\beta}$. Our description of the tire tracks of a bicycle will be in terms of this angle $\Theta$. In fact, we will require that $\Theta$ be signed and satisfy $-\frac{\pi}{2} \leq$ $\Theta \leq \frac{\pi}{2}$, with a positive angle representing a left turn and a negative angle representing a right turn.

The quantification of left and right in terms of mathematics is accomplished by using the principal unit normal vector of a plane curve. The principal unit normal of a plane curve $c$ is defined to be the unit vector $\mathbf{N}$ orthogonal to the unit tangent vector $\mathbf{T}$ such that the ordered pair of vectors $[\mathbf{T}, \mathbf{N}]$ is a rotation of the standard basis vectors $[\mathbf{i}, \mathbf{j}]$ of the plane. This means that if $\mathbf{T}$ is given by $\mathbf{T}=\cos (\phi) \mathbf{i}+\sin (\phi) \mathbf{j}$, then $\mathbf{N}$ is given as $\mathbf{T}$ rotated by $\frac{\pi}{2}$ radians, i.e.,

$$
\mathbf{N}=\cos \left(\phi+\frac{\pi}{2}\right) \mathbf{i}+\sin \left(\phi+\frac{\pi}{2}\right) \mathbf{j}=-\sin (\phi) \mathbf{i}+\cos (\phi) \mathbf{j} .
$$

The vector $\mathbf{N}$ can also be obtained by the standard trick for obtaining a vector orthogonal to $\mathbf{T}=a \mathbf{i}+b \mathbf{j}$ by $\mathbf{N}=-b \mathbf{i}+a \mathbf{j}$. 
Our goal in the remainder of this section is to derive equations for describing the geometry of the bicycle tracks using $\Theta$, and the vectors $\left[\mathbf{T}_{\alpha}, \mathbf{N}_{\alpha}\right]$ and $\left[\mathbf{T}_{\beta}, \mathbf{N}_{\beta}\right]$ associated with a bicycle. In fact, one use of the angle $\Theta$ is to convert between the vectors $\left[\mathbf{T}_{\alpha}, \mathbf{N}_{\alpha}\right]$ and $\left[\mathbf{T}_{\beta}, \mathbf{N}_{\beta}\right]$, see Figure 4,

$$
\begin{aligned}
& \mathbf{T}_{\alpha}=\cos \Theta \mathbf{T}_{\beta}+\sin \Theta \mathbf{N}_{\beta} \quad \mathbf{T}_{\beta}=\cos \Theta \mathbf{T}_{\alpha}-\sin \Theta \mathbf{N}_{\alpha} \\
& \text { and } \\
& \mathbf{N}_{\alpha}=-\sin \Theta \mathbf{T}_{\beta}+\cos \Theta \mathbf{N}_{\beta} \quad \mathbf{N}_{\beta}=\sin \Theta \mathbf{T}_{\alpha}+\cos \Theta \mathbf{N}_{\alpha}
\end{aligned}
$$

Combining (1) and (4), we have

$$
\beta=\alpha-\left(\cos \Theta \mathbf{T}_{\alpha}-\sin \Theta \mathbf{N}_{\alpha}\right),
$$

which gives the back tire's position in terms of the front tire's position and direction.

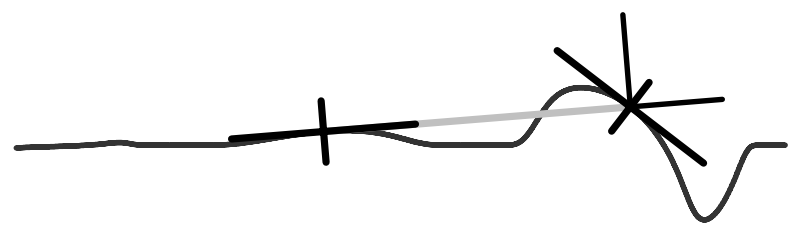

Figure 4. Relations between $\left[\mathbf{T}_{\alpha}, \mathbf{N}_{\alpha}\right]$ and $\left[\mathbf{T}_{\beta}, \mathbf{N}_{\beta}\right]$

The equations (1) and (5) allow one to determine the one tire track given the other tire track. This is useful for determining which direction the bicycle was travelling, as these equations provide a simple physical check (see [9, p. 63]), much like the physical check previously described. However, these equations by themselves do not permit us to mathematically describe the creation of a bicycle track. In order to do this, we need derive a system of differential equations for creating the tire tracks of a bicycle. We will provide a geometric approach to derive these equations. An alternate approach using systems of Ricatti type equations is given in [3], see also [7]. The key fact upon which our derivation relies is the remarkable fact that a plane curve is uniquely described, up to a rotation and translation, by its signed curvature. For completeness, we include a brief description of this remarkable fact, known as the fundamental theorem of plane curves, see [2], [8] or [10] for more details.

The fundamental theorem of plane curves is a consequence of how the unit tangent vector $\mathbf{T}$ of the curve and the principal unit normal vector $\mathbf{N}$ change with respect to the parameter of the curve. In fact, it is an exercise in vector calculus [11, p. 725, Ex. 45, 46] to derive the Frenet frame equations,

$$
\frac{d \mathbf{T}}{d s}=\kappa \mathbf{N} \quad \text { and } \quad \frac{d \mathbf{N}}{d s}=-\kappa \mathbf{T},
$$

from the fact that $\mathbf{T}$ and $\mathbf{N}$ are orthogonal unit vectors, where $\kappa$ is the signed curvature of the curve and $s$ is its arclength. A curve $\mathbf{c}$ with signed curvature $\kappa$ is given by solving the Frenet frame equations (6) as follows. First, write the unit tangent vector $\mathbf{T}$ as $\mathbf{T}=\cos (\phi) \mathbf{i}+\sin (\phi) \mathbf{j}$, so the signed curvature is given by $\kappa=d \phi / d s$. Then, a curve $c$ with signed curvature $\kappa$ is given by

$$
\mathbf{c}=\int(\cos (\phi) \mathbf{i}+\sin (\phi) \mathbf{j}) d s \quad \text { where } \quad \phi=\int \kappa d s .
$$


In (7), we have suppressed information in the integral signs for convenience. We should have used definite integrals and initial conditions rather than indefinite integrals.

The principal advantage of using fundamental theorem of plane curves is that if we know how the curvature of the track evolves with time or arclength then we can construct the bicycle track by solving the Frenet frame equations. By differentiating (1) with respect to $t$ and using the chain rule, one can show with the Frenet frame equations that the curvature of the back tire is given by

$$
\kappa_{\beta}=\tan \Theta,
$$

and the speed of the tires are related by

$$
\frac{d s_{\alpha}}{d t}=\sec \Theta \frac{d s_{\beta}}{d t} .
$$

By differentiating the equation for $\mathbf{T}_{\alpha}$ in (4) with respect to $t$, one can show that

$$
\kappa_{\alpha}=\cos \Theta \frac{d \Theta}{d s_{\beta}}+\sin \Theta \quad \text { or } \quad \kappa_{\alpha}=\frac{d \Theta}{d s_{\alpha}}+\sin \Theta .
$$

The details of these calculation are shown in [6]. Notice that (8) and (10) allow us to determine both the front tire and the back tire knowing the angle $\Theta$ and the speed of the back tire, since this information allows us to solve the Frenet frame equations for both tire. It is these equations that allow us to create a mathematical bicycle track. These equations also match the everyday experience of riding a bicycle, where we control the path of the bicycle by turning the front tire and controlling the speed of the back tire.

\section{Construction of an initial track segment}

Once we have an appropriate initial curve segment, we can use the equations in the last section to generate the tire tracks. In this section, we show how the analysis also provides us with conditions on the initial segment, and that there are initial segments for which we can start our construction method. We again want to emphasize that this is the hard part in our construction, as we need the initial curve segment to satisfy an infinite number of compatibility conditions.

If we suppose that $\gamma$ is a unicycle track that can be created with a bicycle, then there are continuous functions $s_{1}(t)$ and $s_{2}(t)$ such that $\beta(t)=\gamma\left(s_{1}(t)\right)$ and $\alpha(t)=\gamma\left(s_{2}(t)\right)$. This allows us to rewrite (1) and (4) respectively in terms of $\gamma$ as

$$
\gamma\left(s_{2}\right)=\gamma\left(s_{1}\right)+\mathbf{T}_{\gamma}\left(s_{1}\right)
$$

and

$$
\mathbf{T}_{\gamma}\left(s_{2}\right)=\frac{1}{\sqrt{1+\left(\kappa_{\gamma}\left(s_{1}\right)\right)^{2}}} \mathbf{T}_{\gamma}\left(s_{1}\right)+\frac{\kappa_{\gamma}\left(s_{1}\right)}{\sqrt{1+\left(\kappa_{\gamma}\left(s_{1}\right)\right)^{2}}} \mathbf{N}_{\gamma}\left(s_{1}\right) .
$$

Furthermore, by differentiating (1) with respect to time and converting derivatives with respect to time to derivatives with respect to arclength, we get

$$
\kappa_{\gamma}\left(s_{2}\right)=\left.\frac{1}{\left(\sqrt{1+\left(\kappa_{\gamma}\left(s_{1}\right)\right)^{2}}\right)^{3}} \frac{d \kappa_{\gamma}}{d s}\right|_{s=s_{1}}+\frac{\kappa_{\gamma}\left(s_{1}\right)}{\sqrt{1+\left(\kappa_{\gamma}\left(s_{1}\right)\right)^{2}}} .
$$


Notice that (13) defines the signed curvature $\kappa$ of $\gamma$ when $s=s_{2}$ in terms of the signed curvature $\kappa$ and the derivative of the signed curvature with respect to arclength when $s=s_{1}$. Therefore, we find that the derivatives of the curvature $\kappa$ with respect to arclength when $s=s_{2}$ are given in terms of the curvature and the derivatives of the curvature with respect to arc-length when $s=s_{1}$, that is

$$
\frac{d^{n} \kappa}{d s^{n}}\left(s_{2}\right)=F_{n}\left(k\left(s_{1}\right), \frac{d \kappa}{d s}\left(s_{1}\right), \ldots, \frac{d \kappa^{(n+1)}}{d s^{n+1}}\left(s_{1}\right)\right),
$$

for specific functions $F_{n}$ that can be determined by repeatedly differentiating (13). This establishes an infinite number of compatibility conditions on any initial curve segment that can be used in our construction process.

The question then is: Does there exist a curve segment $\gamma(s)$ that satisfies (11), (12), (13) and (14)?

Luckily, it is easy to verify that a straight line $\left(\kappa_{\gamma} \equiv 0\right)$ satisfies these compatibility conditions. The important observation, based on the local nature of our compatibility conditions, is that we only need a curve whose curvature and all its derivatives are zero at the two endpoint of the initial segment. This requirement is equivalent to finding a non-constant function all of whose derivatives are equal to zero at two points. Such functions exist, but they are outside the realm of analytic functions one normally encounters in calculus. For example, all the derivatives of

$$
f(t)= \begin{cases}0 & \text { if } t=0 \\ \mathrm{e}^{-1 / x^{2}} & \text { if } t \neq 0\end{cases}
$$

when $t=0$ are zero, but the function is not constant (see [1] for more details).

Using functions like (15), we can define a class of infinitely differentiable functions that are identically equal to zero when $t<0$ and when $t>1$ but are not equal to zero in the interval $0<t<1$. Choose a smooth function $h(t)$ on $\mathbb{R}$ with $h(0)=h(1)=0$ and $h(t)>0$ for $0<t<1$, for instance take $h(t)=\sin ^{2}(\pi t)$, and let

$$
\varphi(t)= \begin{cases}0 & \text { if } t \leq 0 \\ \mathrm{e}^{-1 / h(t)} & \text { if } 0<t<1 \\ 0 & \text { if } t \geq 1\end{cases}
$$

The same type of calculation that shows $f(t)$ in (15) is infinitely differentiable, also shows that $\varphi(t)$ is infinitely differentiable and all its derivatives when $t=0$ and when $t=1$ are zero.

We can now define an initial curve for generating a unicycle track with a bicycle. Start with a parametric equation of a line $\mathbf{L}(t)$, and choose a smooth function $\varphi$ so that $\varphi(t)=0$ for $t \leq 0$ and $t \geq 1$, as in (16). An initial curve segment can then defined by

$$
\gamma(t)=\mathbf{L}(t)+\varphi(t) \mathbf{c}(t) \text { for } 0 \leq t \leq 1
$$

for any smooth parametric curve c. In fact, the initial curve to generate the unicycle track in Figure 1 was constructed using (17) with $h(t)=\sin ^{2}(\pi t)$ in (16), and $\mathbf{L}(t)=[t, 0], \mathbf{c}(t)=\left[t, \frac{1}{4} \sin (t)\right]$.

\section{A numerical construction}

In principle, we can now construct a single tire track with a bicycle from an initial segment of form (17) using our physical construction. We use (11) to inductively define 
the forward direction. The backward direction is harder, as we need to solve a differential difference equation to determine the angle $\Theta$ as we push the bicycle backwards. This differential difference equation is given by equating the curvatures at a point on the unicycle track given from the front tire perspective and the back tire perspective,

$$
\left.\left(\frac{d \Theta}{d s_{\alpha}}+\sin \Theta\right)\right|_{t=t_{1}}=\left.(\tan (\Theta))\right|_{t=t_{2}},
$$

where $t_{1}<t_{2}$ and $\alpha\left(t_{1}\right)=\beta\left(t_{2}\right)$. However, because of the inductive nature of the forward direction and the general difficulties in solving the nonlinear differential difference equation (18), we will use numerical methods in this section to approximate a unicycle track that can be created with a bicycle.

First, we construct an initial segment parameterized with respect to $t$ on the interval $0 \leq t \leq 1$ with the condition that $d \gamma / d t \neq 0$ so the unit tangent vector $\mathbf{T}_{\gamma}$ is welldefined. We then sample the curve at $M+1$ points, for instance set $t_{i}=i / M$ with $i=$ $0,1,2, \ldots, M$, and define $\mathbf{c}_{i}=\gamma\left(t_{i}\right)$. Symbolically (or numerically), we then calculate the unit tangent vector $\mathbf{T}_{i}$ and the curvature $\kappa_{i}$ for each sampled point $\mathbf{c}_{i}$. Thus, we have $M+1$ data sets $\left(\mathbf{c}_{i}, \mathbf{T}_{i}, \kappa_{i}\right)$ from which we can obtain an approximation of the initial curve segment by interpolation. In our calculation, we will also use the principal unit normal vector $\mathbf{N}_{i}$ and the angle $\Theta_{i}$, which can be computed from $\mathbf{T}_{i}$ and $\kappa_{i}$ using $\Theta_{i}=\arctan \left(\kappa_{i}\right)$ and $\mathbf{N}_{i}=-b \mathbf{i}+a \mathbf{j}$ if $\mathbf{T}_{i}=a \mathbf{i}+b \mathbf{j}$.

To produce the forward direction of the unicycle track, we set $\beta\left(t_{i}\right)=\mathbf{c}_{i}$, and use the equations (1), (4) and (10) to define $\left(\mathbf{c}_{i}, \mathbf{T}_{i}, \kappa_{i}\right)$ for $i>M$ by setting $\mathbf{c}_{i+M}=\alpha\left(t_{i}\right)$. To calculate $\kappa_{i+M}$, we use finite difference approximation to estimate the derivative in (10). Thus, we have the iterative formulas

$$
\left\{\begin{aligned}
\mathbf{c}_{i+M} & =\mathbf{c}_{i}+\mathbf{T}_{i} \\
\mathbf{T}_{i+M} & =\cos \left(\Theta_{i}\right) \mathbf{T}_{i}+\sin \left(\Theta_{i}\right) \mathbf{N}_{i} \\
\mathbf{N}_{i+M} & =-\sin \left(\Theta_{i}\right) \mathbf{T}_{i}+\cos \left(\Theta_{i}\right) \mathbf{N}_{i} \\
\kappa_{i+M} & =\frac{\Theta_{i+1}-\Theta_{i}}{\left\|\mathbf{c}_{i+1}-\mathbf{c}_{i}\right\|}+\sin \left(\Theta_{i}\right) \\
\Theta_{i+M} & =\arctan \left(\kappa_{i+M}\right)
\end{aligned}\right.
$$

that will produce an extension of the unicycle track by pushing the bicycle forward.

The backward direction requires solving (18) using numerical methods. We use Euler's method for simplicity, though we could use any numerical integration technique. By finite difference approximation in (10), we have

$$
\left.\frac{d \Theta}{d s_{\alpha}}\right|_{t=t_{i}} \approx \frac{\Theta\left(t_{i}\right)-\Theta\left(t_{i-1}\right)}{\left\|\alpha\left(t_{i}\right)-\alpha\left(t_{i-1}\right)\right\|} .
$$

Thus, we can rewrite (18) using (19) as

$$
\Theta_{i-1}=\Theta_{i}+\left(\sin \left(\Theta_{i}\right)-\tan \left(\Theta_{i+M}\right)\right)\left\|\mathbf{c}_{i+M}-\mathbf{c}_{i+M-1}\right\| .
$$

To determine $\mathbf{c}_{i-1}$, we use (5) to calculate the back tire position at time $t$ from the front tire position and direction at time $t$. However, since we also need $\mathbf{T}_{i-1}$ to continue the process, we first use the relation in (4) to calculate $\mathbf{T}_{i-1}$ from $\mathbf{T}_{\alpha}\left(t_{i-1}\right)=\mathbf{T}_{i+M-1}$ and 
$\mathbf{N}_{\alpha}\left(t_{i-1}\right)=\mathbf{N}_{i+M-1}$. Therefore, we have the iterative formulas

$$
\left\{\begin{aligned}
\Theta_{i-1} & =\Theta_{i}+\left(\sin \left(\Theta_{i}\right)-\tan \left(\Theta_{i+M}\right)\right)\left\|\mathbf{c}_{i+M}-\mathbf{c}_{i+M-1}\right\| \\
\kappa_{i-1} & =\tan \left(\Theta_{i-1}\right) \\
\mathbf{T}_{i-1} & =\cos \left(\Theta_{i-1}\right) \mathbf{T}_{i+M-1}-\sin \left(\Theta_{i-1}\right) \mathbf{N}_{i+M-1} \\
\mathbf{N}_{i-1} & =\sin \left(\Theta_{i-1}\right) \mathbf{T}_{i+M-1}+\cos \left(\Theta_{i-1}\right) \mathbf{N}_{i+M-1} \\
\mathbf{c}_{i-1} & =\mathbf{c}_{i+M-1}-\mathbf{T}_{i-1}
\end{aligned}\right.
$$

that will produce an extension of the unicycle track by pushing the bicycle backward.

Using this numerical method and linear interpolation, we created Figure 5 as an extension of the initial segment

$$
\gamma(t)=\left[t, \varphi(t) t^{2}(1-t)^{2}\right]
$$

where $\varphi(t)$ is of the form (16) with $h(t)=\sin ^{2}(\pi t)$.

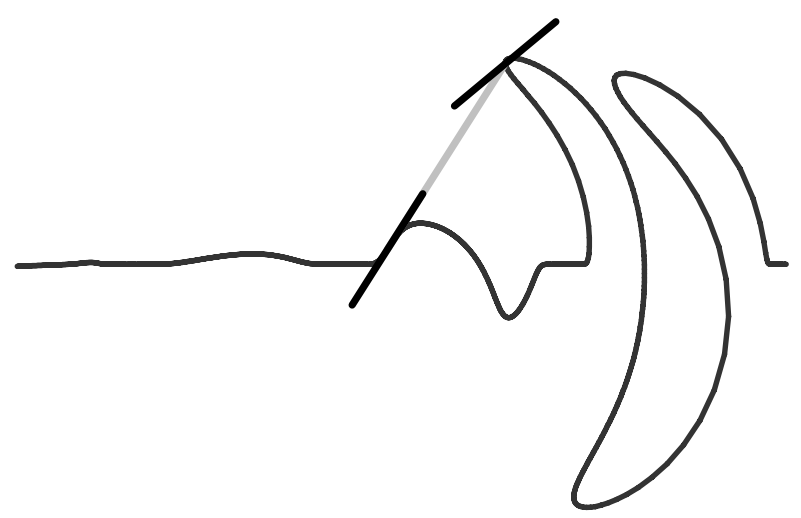

Figure 5. A single tire track that can be created with a bicycle

The potential problem with our numerical algorithm (or any numerical solution) is whether we have convergence as the initial sampling of the segment is refined. For the forward direction, we are guaranteed convergence, because we have a formal extension of the curve by (11). In the backward direction, we can use standard arguments in differential equations to guarantee local convergence, but global convergence is difficult. We will defer that issue to another paper [5], however our numerical method produces curves (through interpolation) that appear to converge at least for some initial curve segments. The main problem in the backward direction is guaranteeing that when we solve the differential difference equation that the angle $\Theta$ satisfies $-\frac{\pi}{2}<\Theta<\frac{\pi}{2}$, so the curvature is continuous and nonsingular.

Another issue that arises once one has looked at some of the curves generated by this algorithm is whether it is possible to physically construct a unicycle track by our methods. We have so far ignored the turning radius of the bicycle, and assumed that $-\frac{\pi}{2}<\Theta<\frac{\pi}{2}$. In actuality, the turning angle is restricted by $-\epsilon<\Theta<\epsilon$ for some positive number $\epsilon$ considerably less than $\frac{\pi}{2}$. For all the unicycle tracks generated by our methods, it seems that $\Theta$ gets arbitrarily close to $\pm \frac{\pi}{2}$, which seems to indicate that it is impossible to generate a large section of a unicycle track with a bicycle. However, 
whether or not it is physically possible, we have accomplished one of our goals. We have shown that it is possible to construct a unicycle track with a bicycle. In order to see the construction of such a track, we refer the reader to the web-page [6], where there are several animations of bicycles constructing unicycle tracks, and a Maple worksheet that can be downloaded which implements the numerical construction.

Acknowledgments. The author thanks Jim Tanton for originally asking him question, "Can a bicycle create a unicycle track?". Also, the author thanks Jim Tanton, Stan Wagon, and the reviewers for their comments on the earlier versions of this paper.

\section{References}

1. David Bressoud, A Radical Approach to Real Analysis, Mathematical Association of America, 1994, pp. 5358 and $74-76$.

2. Manfredo P. DoCarmo, Differential Geometry of Curves and Surfaces, Prentice-Hall, 1976.

3. Steven R. Dunbar, Reinier J. Bosman, and Sander E. M. Nooij, The track of a bicycle back tire, Mathematics Magazine 74 (2001) 273-287.

4. C. Henry Edwards and David E. Penney, Calculus, with Analytic Geometry and Early Transcendentals, 5th ed., Prentice-Hall, 1998, Section 12.6, pp. 759-773.

5. D. L. Finn, in preparation.

6. D. L. Finn, http://www.rose-hulman.edu/ finn/research/unicycle.htm

7. H. I. Freedman and S. D. Riemenschneider, Determining the path of the rear wheels of a bus, SIAM Review 25 (1983) pp. 561-567.

8. Alfred Gray, Modern Differential Geometry of Curves and Surfaces, CRC Press, 1993.

9. Joseph D. E. Konhauser, Dan Velleman, and Stan Wagon, Which Way Did the Bicycle Go?, Mathematical Association of America, 1996.

10. John Oprea, Differential Geometry and its Applications, Prentice-Hall, 1997.

11. James Stewart, Calculus, Concepts and Contexts, 2nd ed., Brooks/Cole, 2001, Section 10.3, pp. 717-725.

\section{An Unrealized Hope}

Only by taking infinitesimally small units for observation (the differential of history, that is, the individual tendencies of men) and attaining the art of integrating them (that is, finding the sum of these infinitesimals) can we hope to arrive at the laws of history.

Thus wrote Leo Tolstoy in War and Peace, published in 1865-69. The excerpt can be found in the Norton Critical Edition, 1966, p. 918. 